\title{
Antropometria Clássica e Músculo Adutor do Polegar na Determinação do Prognóstico Nutricional em Pacientes Oncológicos
}

Classical Anthropometry and the Adductor Pollicis Muscle to Determine the Nutritional Prognosis in Patients with Cancer

Antropometría Clásica y Músculo Aductor del Pulgar en la determinación del Pronóstico Nutricional en Pacientes Oncológicos

Betânia de Jesus e Silva de Almendra Freitas', Larissa Coimbra Mesquita², Nayanna de Jesus Vilhena Teive³ ${ }^{3}$ Samilys Ramos de Souza ${ }^{4}$

\section{Resumo}

A avaliação nutricional é amplamente reconhecida por sua importância no acompanhamento hospitalar; direcionamento da conduta médica em todo processo de hospitalização; investigação para corrigir deficiências nutricionais; e ferramenta para fins prognósticos. A apatia induzida pela desnutrição provoca redução das atividades diárias resultando em diminuição progressiva da espessura do músculo adutor do polegar. O objetivo do trabalho foi estabelecer o prognóstico nutricional, por meio da antropometria clássica e espessura do músculo adutor do polegar, em pacientes oncológicos. O estudo realizado foi transversal de campo do tipo quantitativo. Foram avaliados 82 pacientes oncológicos, adultos e idosos, internados no Hospital São Marcos em Teresina (PI). Os pacientes foram submetidos à avaliação nutricional pela antropometria clássica (\%PP, CB, CMB, $\mathrm{AMBc}$ e $\mathrm{PCT}$ ) e a medida da espessura do músculo adutor do polegar. O estado nutricional dos pacientes caracterizou-se por perda grave de peso $(56,1 \%)$, preservação de massa muscular e prevalência de perda grave de massa gorda $(25,93 \%)$. A média da espessura do músculo adutor do polegar para o total da amostra foi de $13 \pm 3,2 \mathrm{~mm}$. O músculo adutor do polegar não se associou ao tempo de internaçáo e mortalidade. $\mathrm{O}$ \%PP foi o parâmetro que melhor se correlacionou com o tempo de internação ( $\mathrm{p}=0,007)$. Os demais parâmetros não se associaram a nenhum dos indicadores de morbi-mortalidade estudados. A espessura do músculo adutor do polegar não se revelou um bom indicador prognóstico antropométrico, podendo essa afirmação ser atribuída à presença de um menor comprometimento do estado nutricional observada na maioria dos pacientes e por permanecerem menos tempo no ambiente hospitalar.

Palavras-chave: Pacientes Internados; Avaliação Nutricional; Antropometria; Estudos Transversais; Análise Quantitativa; Teresina (PI)

Faculdade de Saúde, Ciências Humanas e Tecnológicas do Piauí (NOVAFAPI).

Hospital São Marcos (Associação Piauiense de Combate ao Câncer).

${ }^{1}$ Mestre em Ciências e Saúde pela Universidade Federal do Piaú (UFPI). Professora Adjunta do Departamento de Nutrição da UFPI e NOVAFAPI, Brasil.

${ }^{2}$ Nutricionista. Professora do Curso Técnico de Enfermagem do Centro de Educação Profissional Nossa Senhora das Graças. Caxias (MA), Brasil.

${ }^{3}$ Mestranda em Atividade Física e Saúde/Rede Euroamericana de Motricidade Humana. Professora do Curso de Nutrição da Faculdade NOVAFAPI, Brasil.

${ }^{4}$ Nutricionista. Secretaria Municipal de Saúde de Croatá (CE), Brasil. Secretaria Municipal de Saúde de Ibiapina (CE), Brasil.

Endereço para correspondência: Betânia de Jesus e Silva de Almendra Freitas. Campus Ministro Petrônio Portela UFPI. Departamento de Nutrição SG 13/UFPI. Teresina (PI), Brasil. CEP: 64049-550.E-mail: betaniafreitas2004@hotmail.com.br 


\section{INTRODUÇÃO}

O câncer é uma enfermidade multicausal crônica, caracterizada pelo aumento da replicação de células anormais até a formaçáo de uma massa de tecido, o tumor $^{1}$.

No Brasil, o câncer é a segunda causa de morte por doença, apenas superada pelas doenças cardiovasculares. De acordo com o Ministério da Saúde, as estimativas, para o ano de 2010, válidas para o ano de 2011, apontam que ocorrerão 489.270 novos casos de câncer (236.240 no sexo masculino e 253.030 no sexo feminino) ${ }^{2}$.

No geral, os pacientes portadores de neoplasia maligna apresentam-se com alto risco nutricional, em decorrência das alteraçóes metabólicas oriundas da patologia e dos tipos de tratamentos utilizados ${ }^{3}$. As consequências da desnutriçáo nesses pacientes sáo: maior tempo de internaçáo; aumento das taxas de morbidade e mortalidade; complicaçóes infecciosas e complicaçôes secundárias; e maiores custos para o sistema de saúde. Estando ainda associada à diminuição da eficácia do tratamento especifico, à redução da qualidade de vida e à mudança da autoimagem, com maiores riscos de infecções pós-operatórias ${ }^{4,5}$.

A incidência de desnutrição em pacientes oncológicos é de $30 \%$ a $50 \%$ dos casos, podendo levar à caquexia, a qual é uma das causas mais comuns de óbitos entre esses pacientes e está presente em cerca de $80 \%$ na fase final de suas vidas ${ }^{6}$.

Segundo o estudo Inquérito Brasileiro de Avaliação Nutricional (IBRANUTRI) com 4.000 doentes hospitalizados na rede pública, distribuídos em 25 hospitais de 12 Estados brasileiros e no Distrito Federal, foi identificado que $20,1 \%$ dos pacientes internados eram portadores de câncer. Destes, 66,4\% apresentavam-se com desnutrição, sendo $45,1 \%$ de grau moderado e $21,3 \%$, grave ${ }^{7}$.

A avaliação nutricional é amplamente reconhecida por sua importância no acompanhamento hospitalar, direcionamento da conduta médica em todo processo de hospitalização e investigação para corrigir deficiências nutricionais. Portanto, o objetivo da investigação do estado nutricional, além de detectar situaçóes de risco nutricional, contribui para o planejamento dos cuidados médicos e representa uma ferramenta para fins prognósticos ${ }^{8}$.

Apesar da importância da avaliação muscular na caracterização do estado nutricional, ainda permanecem limitados os métodos para sua aferição direta ${ }^{9}$, porém o músculo adutor do polegar (MAP) é o único músculo que permite uma adequada avaliação de sua espessura, por apresentar-se anatomicamente bem definido, ser plano e estar situado entre duas estruturas ósseas e, principalmente, por ser reprodutível por outros pesquisadores. Logo, a espessura do MAP trata-se de uma medida direta, não havendo necessidade de aplicaçáo de fórmulas para o cálculo de seu valor real ${ }^{10}$.

A atrofia do MAP reflete a perda da vida laborativa. A apatia induzida pela desnutrição provoca redução das atividades diárias resultando em diminuição progressiva da espessura do MAP. Assim, a sua espessura pode ser utilizada como um parâmetro antropométrico preditor para a evolução clínica de pacientes, incluindo os oncológicos $^{10}$.

Segundo Cabral \& Correia $^{11}$, o estabelecimento do prognóstico do paciente pode guiar melhor a avaliação da terapia nutricional, sendo fundamental para tomar decisōes no tratamento do paciente com câncer, de forma que deve ser realizado o mais precocemente possível.

Tendo em vista que o prognóstico de pacientes portadores de câncer é de difícil mensuração e por esses pacientes apresentarem maior risco nutricional, o objetivo do presente estudo foi estabelecer o prognóstico nutricional, por meio da antropometria clássica e espessura do MAP, em pacientes oncológicos.

\section{MATERIAIS E MÉTODOS}

O presente estudo foi transversal, de campo do tipo quantitativo, realizado no Hospital Sáo Marcos (Associação Piauiense de Combate ao Câncer), Teresina (PI), Brasil. A pesquisa foi analisada e aprovada pelo Comitê de Ética em Pesquisa do Hospital São Marcos, parecer de 01.009/2008.

De certo, além da vontade expressa de participar do estudo, a amostra totalizou 82 pacientes selecionados por demanda espontânea, os quais contemplaram os critérios de inclusão adotados, a saber: maiores de 18 anos, internados, em acompanhamento regular no Serviço, e que aceitaram participar da pesquisa mediante assinatura do Termo de Consentimento Livre e Esclarecido.

Os critérios de exclusáo adotados foram: pacientes portadores de patologias que pudessem exercer influência negativa no trofismo muscular localizado ou geral, exceto desnutrição per si e/ou alteraçôes relacionadas à patologia de base; pacientes com alteração do nível de consciência, na ausência de acompanhante; pacientes com qualquer grau de edema que possa interferir na realizaçáo das medidas e pacientes alimentados exclusivamente através de cateteres ou ostomias.

Os pacientes foram avaliados pelos parâmetros antropometria clássica e medida da espessura do MAP durante o período de internaçáo.

A antropometria clássica constou de: percentual de perda de peso $(\% \mathrm{PP})$, circunferência do braço $(\mathrm{CB})$, prega 
cutânea tricipital (PCT), circunferência muscular do braço $(\mathrm{CMB})$ e área muscular do braço corrigida ( $\mathrm{AMBc}$ ).

O peso corpóreo dos pacientes capazes de deambular foi aferido em uma balança digital Plenna ${ }^{\circ}$, com capacidade de $180 \mathrm{~kg}$. Em pacientes acamados, o peso foi estimado, indiretamente, por meio da fórmula desenvolvida por Chumlea et al. ${ }^{12}$. O peso usual foi informado pelo paciente. $\mathrm{O} \% \mathrm{PP}$ permitiu a classificação do estado nutricional segundo Blackburn et al. ${ }^{13}$.

Para a obtenção do $\mathrm{CB}$, utilizou-se uma fita métrica não flexível. A PCT foi aferida com o adipômetro de Lange, exercendo uma pressão contínua de $10 \mathrm{~g} / \mathrm{mm}^{2}$. A CMB foi obtida a partir dos valores de CB e PCT, por meio de fórmula. $\mathrm{Na}$ obtenção da $\mathrm{AMBc}$, utilizaram-se fórmulas de acordo com o gênero. As adequaçôes de $\mathrm{CB}$, PCT e CMB foram realizadas segundo valores de referência estabelecidos por Jelliffe ${ }^{14}$ para adultos e por Kuczmarski et al. ${ }^{15}$ para pacientes acima de 60 anos. Para $\mathrm{AMBc}$, os valores de referência utilizados foram os estabelecidos por Frisancho ${ }^{16}$ para adultos e Menezes \& Marucci ${ }^{17}$, para idosos.

A medida da musculatura adutora do polegar foi realizada no lado dominante. Durante a aferição, o indivíduo estava sentado com a face ventral da mão repousando sobre o joelho e o cotovelo em ângulo de aproximadamente $90^{\circ}$ sobre o membro inferior homolateral. Utilizou-se o adipômetro de Lange, exercendo uma pressão contínua de $10 \mathrm{~g} / \mathrm{mm}^{2}$ para pinçar o músculo adutor no vértice de um triângulo imaginário formado pela extensão do polegar e do indicador. A média de três medidas consecutivas foi utilizada como medida final ${ }^{18}$.

O tempo de internação e a evolução clínica dos pacientes avaliados (alta ou óbito) foram obtidos por meio dos dados registrados em seus prontuários médicos após um período de dois meses da realização da avaliação do estado nutricional.
Para a análise estatística dos dados, utilizou-se o software estatístico SPSS 13,0 for Windows ${ }^{\circ}$. O critério de significância foi o nível de $5 \%$, ou seja, o valor de $\mathrm{p} \leq 0,05$. Para variáveis numéricas, foi utilizado o coeficiente de correlação de Spearman para medir o grau de associação entre os parâmetros. Utilizou-se também o teste qui-quadrado para correlaçôes simples, avaliando sua significância pelo método de Pearson. Para os desfechos categóricos (mortalidade), utilizou-se a área sobre a curva ROC.

\section{RESULTADOS}

Participaram do estudo 82 pacientes adultos e idosos, portadores de câncer, dentre os quais, $62,2 \%$ pertenciam ao sexo feminino e $37,8 \%$ ao sexo masculino. A média de idade dos pacientes foi de 49,7 anos $\pm 16,1$ anos. A Tabela 1 apresenta a análise descritiva das variáveis quantitativas estudadas e tem por objetivo traçar o perfil geral dessa amostra.

Foram diagnosticados 33 tipos de câncer: 15 (18,29\%) pacientes apresentaram câncer do colo do útero, nove $(10,98 \%)$ leucemia aguda, sete $(8,54 \%)$ câncer de mama e sete $(8,54 \%)$ câncer de reto. Procedendo-se à distribuição dos tipos de câncer por sexo, observou-se que o câncer do colo do útero, seguido de câncer de mama, foram os mais predominantes na populaçấo feminina, representando aproximadamente $30 \%$ e $10 \%$ da amostra, respectivamente. Já na população masculina, o tipo mais prevalente foi o câncer de reto representando aproximadamente $20 \%$ da amostra e leucemia aguda com um percentual de $15 \%$. A leucemia aguda apresentou-se mais prevalente na faixa etária de 18 a 26 anos.

Nos 82 pacientes que compunham a amostra, observou-se que a média da espessura do MAP foi de $13 \pm 3,2 \mathrm{~mm}$, sendo a média desse parâmetro maior na população masculina $(13,6 \mathrm{~mm})$ do que na feminina $(12,7 \mathrm{~mm})$. No que diz respeito à faixa etária, a espessura

Tabela 1. Distribuição das variáveis quantitativas dos pacientes oncológicos em tratamento no HSM. Teresina (PI), 2009

\begin{tabular}{l|c|c|c|c|c|c}
\hline & N & Média & Mediana & Desvio-padrão & Mínimo & Máximo \\
\hline Idade (anos) & 82 & 49,7 & 51,5 & 16,1 & 20 & 89 \\
\hline$\%$ PP & 56 & 11,3 & 8,4 & 10,0 & 1,0 & 46,1 \\
\hline PCT (mm) & 81 & 14,4 & 13,3 & 7,3 & 3,3 & 36,6 \\
\hline CB (cm) & 82 & 26,7 & 26,0 & 4,5 & 16,0 & 37,0 \\
\hline CMB (cm) & 81 & 22,3 & 22,3 & 3,2 & 14,7 & 31,4 \\
\hline AMBc (cm $\left.{ }^{2}\right)$ & 81 & 32,3 & 31,3 & 11,4 & 10,7 & 68,5 \\
\hline Espessura do MAP (mm) & 82 & 13,0 & 12,6 & 3,2 & 6,0 & 22,0 \\
\hline Tempo de internação (dias) & 82 & 9,0 & 5,0 & 12,7 & 2 & 98 \\
\hline
\end{tabular}


do MAP mostrou-se maior em faixas etárias menores do que 58 anos, preservando-se esta na faixa etária acima de 65 anos, para então reduzi-la na faixa 82-90 anos.

Quanto ao \%PP, foi possível determiná-lo em apenas 56 pacientes; dentre estes, a classificação do estado nutricional ocorreu em 41 dos pacientes, uma vez que eles souberam informar o período em que apresentavam o peso usual referido. A média do \%PP da amostra foi de $11,3 \% \pm 10 \%$.

Classificando essa populaçáo nutricionalmente segundo o \%PP, observou-se que 56,10\% (23) dos pacientes apresentaram perda grave de peso e $4,88 \%$ apresentaram perda significativa de peso, conforme Gráfico 1.

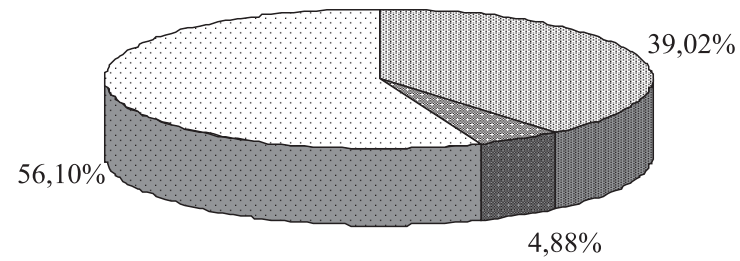

橉 Perda não significativa Perda significativa $\square$ Perda grave

Gráfico 1. Classificação do estado nutricional segundo \%PP dos pacientes oncológicos em tratamento no HSM. Teresina (PI), 2009

As médias gerais da população para PCT, $\mathrm{CB}, \mathrm{CMB}$ e $A M B c$ foram $14,4 \pm 7,3 \mathrm{~mm}, 26,7 \pm 4,5 \mathrm{~cm}, 22,3 \pm 3,2 \mathrm{~cm}$, $32,3 \pm 11,4 \mathrm{~cm}^{2}$, respectivamente.

$\mathrm{Na}$ determinação do estado nutricional de acordo com $\mathrm{CB}, \mathrm{CMB}, \mathrm{AMBc}$, constatou-se que a maioria dos pacientes apresentou-se eutrófico $(40,24 \%, 55,55 \%$, $53,10 \%$ ), respectivamente, conforme Gráficos 2 e 3.

Em relação ao estado nutricional, segundo PCT, revelou-se um quadro de risco nutricional, haja vista que se evidenciou prevalência de desnutrição grave em 25,93\% da amostra, seguida de obesidade em 23,46\% (Gráfico 2).

O tempo médio de internação dos pacientes foi de $9 \pm 12,7$ dias.

A espessura do MAP não apresentou correlação estatística $(p=0,107)$ com o tempo de internação hospitalar dos pacientes oncológicos. Os parâmetros antropométricos clássicos também não apresentaram essa correlação, exceto o \% PP ( $\mathrm{p}=0,007)$, demonstrando que, quanto mais grave a perda de peso, mais tempo o paciente permanece internado.

Durante o período de internação, faleceram 10,98\% (9) dos pacientes e nenhum dos parâmetros estudados se associou com a mortalidade.
Embora a área sobre a curva de ROC do \%PP tenha se mostrado elevada $(0,695)$, evidenciando uma associação com a mortalidade, não houve significância estatística $(\mathrm{p}=0,079)$.

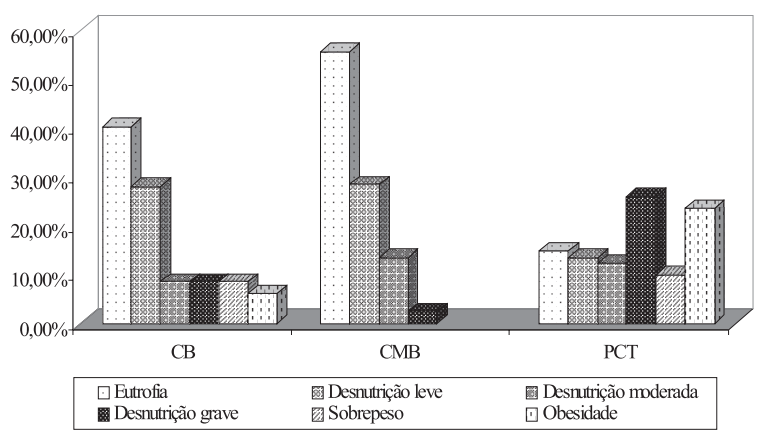

Gráfico 2. Classificação do estado nutricional segundo $C B, C M B$ e PCT dos pacientes oncológicos em tratamento no HSM. Teresina (PI), 2009

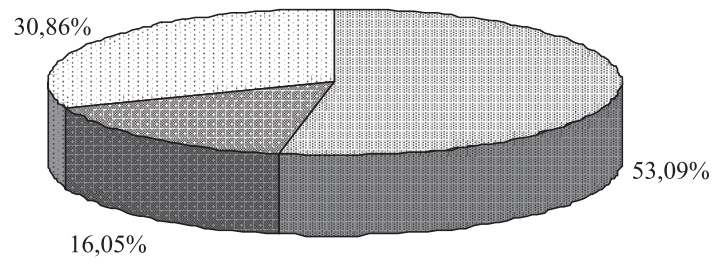

粗 Eutrofia 圆 Denutrição leve/moderada $\square$ Denutrição grave

Gráfico 3. Classificação do estado nutricional segundo $A M B c$ dos pacientes oncológicos em tratamento no HSM. Teresina (PI), 2009

\section{DISCUSSÃO}

O câncer e o estado nutricional estão intimamente relacionados, de modo que alteraçóes fisiológicas oriundas da patologia podem causar desnutriçáo; que, por sua vez, podem influir negativamente na evolução da enfermidade e eficácia do tratamento ${ }^{19}$.

A desnutrição interfere em quase todos os órgãos e/ ou sistemas do corpo humano. Essa afirmação justifica a relação entre desnutrição hospitalar e aumento da morbimortalidade, tempo de internação e custos ${ }^{20}$.

O \%PP é um método antropométrico de suma importância em oncologia, sendo esse parâmetro uma referência à mudança de peso em relaçáo ao peso habitual e sua evolução ao longo do tempo ${ }^{19}$.

A perda involuntária de $10 \%$ ou mais do peso usual nos últimos seis meses significa um substancial comprometimento nutricional e tem sido considerada como um indicador de risco nutricional e de mau 
prognóstico cirúrgico, uma vez que o organismo tendo sofrido um déficit nutricional agudo não consegue desenvolver mecanismos de adaptaçáo imediatos. A avaliaçáo da perda de peso recente parece ser mais sensível na detecçáo de risco nutricional ou mesmo de um quadro de desnutrição aguda ${ }^{21,22}$.

Observou-se neste estudo que 56,1\% da amostra apresentaram perda grave de peso; resultado que se coadunou com trabalho realizado por Diestel et al. ${ }^{22}$, que evidenciou perda de peso significativa ou severa em mais da metade da populaçáo estudada $(55,63 \%)$. Corroborando ainda o estudo conduzido por Alves et $a l .^{23}$, que observaram perda grave de peso em $26,9 \%$ da sua amostra. É possível evidenciar o relevante desgaste nutricional sofrido por tais pacientes, em face do hipercatabolismo imposto pela patologia e tratamento.

A avaliaçáo por parâmetros antropométricos clássicos reflete o grau de desnutrição, porém as alterações nesses parâmetros só surgem após um período variável de desnutrição. Desse modo, todas as modificaçôes somáticas, embora importantes para o diagnóstico de desnutrição crônica, não são capazes de avaliar essa condição na fase $\operatorname{aguda}^{10}$.

A respeito dos métodos para avaliar o estado nutricional, faz-se necessária a utilização das pregas cutâneas e circunferências. Apesar de suas limitaçôes, as pregas cutâneas são métodos práticos para estimar a gordura corpórea e sua distribuição em vários segmentos do corpo. O PCT é o mais amplamente utilizado e avalia a massa gorda periférica ${ }^{5,21}$.

As circunferências são medidas musculares feitas para obter uma estimativa da quantidade e da taxa de variação da proteína muscular esquelética. A CB estima a proteína muscular esquelética total e representa a somatória das áreas constituídas pelo tecido ósseo, muscular e gorduroso do braço, sendo possível derivar a CMB por meio de equaçóes matemáticas que avaliam a reserva de tecido muscular sem correção da área óssea. A AMBc reflete mais adequadamente as mudanças do tecido muscular, uma vez que avalia a reserva do tecido muscular corrigido da área óssea ${ }^{24}$.

Dock-Nascimento et al. ${ }^{4}$, objetivando correlacionar as medidas estimadas de peso e altura com medidas reais em pacientes oncológicos, observaram valores da mediana de PCT e CB de $12,1 \mathrm{~mm}$ e $27,2 \mathrm{~cm}$, respectivamente, valores semelhantes ao encontrado no presente estudo.

Conforme estudo de Ulsenheimer et al. ${ }^{5}$, ao avaliarem o perfil nutricional de pacientes com câncer segundo diferentes indicadores, demonstrou-se a incidência de desnutrição de $66,67 \%$ para $\mathrm{PCT}, 38,89 \%$ para $\mathrm{CB}$ e $16,67 \%$ para CMB. No presente estudo, os percentuais de pacientes desnutridos foram de $51,9 \%$ para PCT,
$45,1 \%$ para $\mathrm{CB}$ e $44,5 \%$ para $\mathrm{CMB}$, indicando uma maior mobilização de massa magra nos pacientes avaliados.

Em relação ao PCT, Alves et al. ${ }^{23}$ constataram que $25,9 \%$ dos doentes tinham PCT abaixo do percentil 5 $(\mathrm{n}=135)$, condizente com o deste trabalho que revelou $25,93 \%$ com desnutrição grave. Entretanto, no mesmo estudo, $58 \%$ dos pacientes apresentavam CMB abaixo do percentil $5(\mathrm{n}=131)$, resultado diferente do encontrado neste estudo, em que mais de $50 \%$ da população apresentaram-se com eutrofia, segundo esse parâmetro.

A avaliação do estado nutricional pelo PCT, por outro lado, apresentou resultados compatíveis com a depleção de massa gorda em $25,93 \%$ da amostra, ressaltando a maior especificidade desse parâmetro antropométrico quando se pretende dimensionar o impacto da perda de massa gorda desencadeada pela doença.

Diferente dos resultados do presente estudo, Andrade $\&$ Lameu $^{10}$ alcançaram diferença estatística significativa quando correlacionaram a espessura do MAP da mão dominante com o tempo de internação $(\mathrm{p}=0,028)$, demonstrando que quanto menor a espessura do MAP mais tempo o paciente permanece internado. Com relação ao \%PP, não observaram correlação estatística $(\mathrm{p}=0,102)$; porém, semelhante ao presente estudo, os autores Andrade $\&$ Lameu $^{10}$ não observaram correlação entre o tempo de internação com CMB ( $\mathrm{p}=0,181)$ e AMB ( $\mathrm{p}=0,735)$.

No que diz respeito ao indicador de mortalidade, o resultado deste trabalho corrobora o resultado da pesquisa conduzida por Andrade \& $\mathrm{Lameu}^{10}$.

No presente estudo, utilizando o valor da mediana de 12,6 mm (cut off $12,6 \mathrm{~mm}$ ), observou-se que 12,82\% dos pacientes que faleceram apresentavam MAP acima do valor da mediana. Logo, não ocorreu associação estatística entre espessura do MAP cut off $12,6 \mathrm{~mm}$ e a mortalidade $(\mathrm{p}=0,611)$; porém Andrade $\& \mathrm{Lameu}^{10}$ demonstraram que $94,7 \%$ dos pacientes com MAP da máo dominante acima do valor da mediana de $14 \mathrm{~mm}(\mathrm{p}=0,028)$ não faleceram durante a internação hospitalar, sugerindo um alto poder preditivo negativo do MAP para mortalidade.

A atrofia do MAP durante a desnutrição não está relacionada com a doença per si, mas com a reduçáo das atividades diárias que ocorrem devido à apatia induzida pela desnutrição e ao elevado tempo de permanência hospitalar, sendo que esses fatores interferem na troficidade muscular e, consequentemente, no valor do MAP. Provavelmente, a inatividade agrava a redução da espessura MAP independentemente do catabolismo e da doença básica do paciente. Assim, não só a redução da massa muscular é um parâmetro prognóstico para complicaçóes no pós-operatório, mas também a perda da função do músculo é um preditor de mortalidade em indivíduos saudáveis ${ }^{10}$. 
Em Andrade \& Lameu $^{10}$, embora as áreas sobre a curva de ROC tenham se mostrado elevadas, não identificaram significância estatística, semelhante aos dados do presente estudo.

Uma perda de peso igual ou superior a $10 \%$ de forma involuntária em um período de tempo de seis meses é um critério de primeira linha para detectar desnutrição, tendo significado prognóstico em pacientes com câncer ${ }^{19}$.

Jamnik et al. ${ }^{25}$ observaram que $66,5 \%$ dos pacientes com sobrevida menor ou igual a um ano tiveram \%PP mais acentuada que os do grupo com sobrevida superior a cinco anos $(35,7 \%)$ e essa diferença foi muito significante $(\mathrm{p}=0,0029)$.

Em estudo realizado com pacientes hospitalizados, sendo $18,2 \%$ desses pacientes portadores de neoplasias malignas, os autores demonstraram que pacientes desnutridos apresentaram aumento significativo da mortalidade (12,4\% vs 4,7\%) e aumento do tempo de permanência hospitalar (média de 16,7 dias vs 10,1 dias) ${ }^{20}$.

Os parâmetros que avaliam massa muscular e massa gorda não demonstraram associação com os valores prognósticos utilizados na pesquisa, porém autores correlacionam a desnutriçáo hospitalar com o aumento da morbidade, mortalidade e tempo de internação; e indicam que o comprometimento do estado nutricional pode ser um dos fatores mais importantes para a interferência da saúde dos pacientes ${ }^{20,8}$.

\section{CONCLUSÃO}

Considerando as variáveis antropométricas estudadas, conclui-se que, apesar de $56,1 \%$ dos pacientes apresentarem perda grave de peso, a maioria da população apresentou-se eutrófica por meio de outros parâmetros antropométricos $(\mathrm{CB}, \mathrm{CMB}, \mathrm{AMBc})$, demonstrando preservação de massa muscular, porém, em relação ao PCT, constatou-se que a maior parte dos pacientes sofreu depleção grave de massa gorda. Concluiu-se; portanto, que, a despeito da perda grave de peso e comprometimento de massa gorda evidenciados, ainda foi possível constatar manutenção da massa muscular na maioria dos pacientes, o que pode sinalizar para um possível excesso de peso no momento do diagnóstico, com imediata depleção de massa adiposa; sabendo-se que a depleção de massa magra é retardada em relação à depleção de massa gorda.

$\mathrm{O} \% \mathrm{PP}$ se correlacionou com o tempo de internaçáo, ou seja, quanto mais grave a perda de peso mais tempo os pacientes permaneceram internados, uma vez que apresentavam mais expressivo déficit nutricional, o que dificultaria a eficácia do tratamento.

Apesar de a espessura no MAP não ter se associado a nenhum indicador prognóstico estudado, é possível inferir-se das consideraçóes acima e dos resultados do estudo que essa espessura reflete à perda da vida laborativa. Podendo atribuir-se a não associação entre a espessura do MAP com o tempo de internaçáo e com mortalidade à presença de um menor comprometimento do estado nutricional, observada na maioria dos pacientes, e por permanecerem menos tempo no ambiente hospitalar, consequentemente, mantendo suas atividades diárias.

Partindo-se do pressuposto que são escassos os estudos utilizando o MAP como um novo indicador prognóstico, faz-se necessária a realização de mais trabalhos envolvendo essa temática em busca de uma melhor compreensão de sua real utilidade na prática clínica.

\section{Declaraçáo de Conflito de Interesses: Nada a Declarar.}

\section{REFERÊNCIAS}

1. Garófolo A, Avesani CM, Camargo KG, Barros ME, Silva SRJ, Taddei JAAC et al. Dieta e câncer: um enfoque epidemiológico. Revista de nutrição da PUCCAMP 2004 out/dez; 17(4): 491-505.

2. Instituto Nacional de Câncer (Brasil). Estimativas 2010: Incidência de Câncer no Brasil. Rio de Janeiro: INCA, 2009.

3. Melo ILP, Dantas MAM, Silva LC, Lima VT, Lima SCVC, Sena KCM. Avaliação nutricional de pacientes cirúrgicos com câncer de cabeça e pescoço sob terapia nutricional enteral. Revista brasileira de nutrição clínica 2006; 21(1):6-11.

4. Dock-Nascimento DB, Aguilar-Nascimento JE, Costa HCBAL, Vale HV, Gava MM. Precisão de métodos de estimativa do peso e altura na avaliação do estado nutricional de pacientes com câncer. Revista brasileira de nutrição clínica 2006; 21(2):111-6.

5. Ulsenheimer A, Silva ACP, Fortuna FV. Perfil nutricional de pacientes com câncer segundo diferentes indicadores de avaliação. Revista brasileira de nutrição clínica 2007; 22(4):299-7.

6. Moreira TR, Moraes CMB. Abordagem nutricional no tratamento e prevenção do melanoma. Revista brasileira de nutrição clínica 2007; 22(4):311-6.

7. Waitzberg DL, Alves CC, Torrinhas RS. Incidência da desnutriçãoo em câncer. In: Waitzberg DL. Dieta, nutriçãao e câncer. São Paulo: Atheneu; 2006. p. 269-76.

8. Merhi VAL, Oliveira MRM, Caran AL, Tristão TMG, Ambo RM, Tanne MA et al. Tiempo de hospitalización y estado nutricional en pacientes hospitalizados. Nutr Hosp 2007; 22(5):590-5.

9. Waitzberg DL, Terra RM. Função muscular e sua relação com nutrição e desnutrição. In: Waitzberg DL. Nutrição oral, enteral e parenteral na prática clínica. 3.ed. São Paulo: Atheneu; 2006. p. 321-5. 
10. Andrade PV, Lameu EB. Espessura do músculo adutor do polegar: um novo indicador prognóstico em pacientes clínicos. Revista brasileira de nutrição clínica 2007;22(1): 28-35.

11. Cabral ELB, Correia MITD. Aspectos nutricionais na abordagem do câncer avançado. In: Waitzberg DL. Dieta, nutrição e câncer. São Paulo: Atheneu; 2006. p. 329-33.

12. Chumlea WC, Roche AF, Steinbaugh ML. Estimating stature from knee height for persons 60 to 90 years of age. J Am Geriatr Soc 1985; 33(2):116-20.

13. Blackburn GL, Bistrian BR, Maini BS, Schlamm HT, Smith MF. Nutritional and metabolic assessment of the hospitalized patient. JPEN J Parenter Enteral Nutr 1977; 1(1):11-22.

14. Jelliffe DB. The assessment of nutritional status of community. Geneve: World Health Organization; 1966. Monograph.

15. Kuczmarski MF, Kuczarisk RJ, Najjar M. Descriptive anthropometric reference data for older Americans. J Am Diet Assoc 2000 Jan; 100(1):59-66.

16. Frisancho AR. Antropometric standards for the assessment of growth and nutritional status. Michigan: The University Academic Press; 1999. p.189.

17. Menezes TN, Marucci MFN. Perfil dos indicadores de gordura e massa muscular corporal dos idosos de Fortaleza, Ceará, Brasil. Cad Saude Publica 2007 dez; 23(12):2887-95.

18. Lameu EB, Gerude MF, Corrêa RC, Lima KA. Adductor Policis Muscle: a new anthropometric parameter. Rev Hosp Clin Fac Med Sao Paulo2004; 59(2):57-62.
19. Cerezo L. Diagnóstico del estado nutricional y su impacto en el tratamiento del câncer. Oncología 2005; 28 (3):129-34.

20 Correia MITD, Waitzberg DL. The impact of malnutrition on morbidity, mortality, length of hospital stay and costs evaluated through a multivariate model analysis. Clinical nutrition 2003; 22(3): 235-9.

21. Rivadeneira DE, Evoy D, Fahey III TJ, Lieberman MD, Daly JM. Nutritional Support of the Cancer Patient. CA Cancer J Clin 1998; 48:69-80.

22. Diestel CF, Horst NL, Sampaio ARD, Mannarino IC, Matzke BD, Simões FC. Percentual de perda de peso comparado com índice de massa corporal (IMC) como fatores de risco nutricional pré-operatório de pacientes oncológicos cirúrgicos. Anais da II Jornada Internacional de Nutrição Oncológica; I Jornada Luso-Brasileira de Nutrição Oncológica. Revista brasileira de cancerologia 2004; 50(4): 351-79.

23. Alves P, Pinto I, Gonçalves F, Costa I, Monteiro C, KentSmith L. Avaliação nutricional de doentes admitidos na unidade de cuidados paliativos do IPOFG-CROP, S.A. Anais da II Jornada Internacional de Nutrição Oncológica; I Jornada Luso-Brasileira de Nutrição Oncológica. Revista brasileira de cancerologia 2004; 50(4): 351-79.

24. Coppini LZ. Avaliação nutricional no paciente com câncer. In: Waitzberg DL. Dieta, nutrição e câncer. São Paulo: Atheneu; 2006. p.385-91.

25. Jamnik S, Santoro IL, Borges EL, Uehara C, Silva VV. Avaliação nutricional em pacientes portadores de câncer de pulmão. Jornal de pneumologia 1998 nov./dez.; 24(6):347-53. 


\begin{abstract}
The nutritional assessment is widely recognized for its importance in medical follow-up, handling of medical procedures throughout the process of hospitalization, investigation in order to correct nutritional deficiencies, and it is a tool for prognosis. Apathy due to malnutrition leads to decreased daily activities, resulting in a progressive decrease in the adductor pollicis muscle thickness. This paper aimed to establish the nutritional prognosis, by means of classical anthropometry and the adductor pollicis muscle thickness, in patients with cancer. This was a quantitative cross-sectional field study. Eighty-two cancer patients, both adult and elderly, admitted in the São Marcos Hospital in Teresina (PI), were evaluated. The patients were submitted to the nutritional assessment through classical anthropometry (\%WC, AC, MAC, CAMA and TCF) and the measure of the adductor pollicis muscle thickness. The patients' nutritional status was characterized by severe weight loss $(56.1 \%)$, preservation of muscle mass and prevalence of severe body fat loss $(25.93 \%)$. The average thickness of the adductor pollicis muscle for the entire sample was $13 \pm 3.2 \mathrm{~mm}$. The adductor pollicis muscle was not associated with the length of hospital stay and mortality. WC was the parameter that best correlated with hospital length of stay $(\mathrm{p}=0.007)$. The other parameters were not associated with any of the morbidity and mortality indicators studied. The thickness of the adductor pollicis muscle was not a good anthropometric prognostic indicator. This may be attributed to less affected nutritional status, as observed in most patients, and shorter length of hospital stay.

Key words: Inpatients; Nutrition Assessment; Anthropometry; Cross-Sectional Studies; Quantitative Analysis; Teresina city
\end{abstract}

\title{
Resumen
}

La evaluación nutricional es ampliamente reconocida por su importancia en el acompańamiento hospitalario; direccionamiento de la conducta médica en todo el proceso de hospitalización; investigación para corregir las deficiencias nutricionales y la herramienta para pronósticos. La apatía inducida por la desnutrición lleva a la reducción de las actividades diarias que resulta en una disminución progresiva de la espesura del músculo aductor del pulgar. El objetivo del trabajo fue establecer el pronóstico nutricional por medio de la antropometría clásica y espesura del músculo aductor del pulgar en pacientes oncológicos. El estudio realizado fue transversal de campo del tipo cuantitativo. Se evaluaron 82 pacientes oncológicos, adultos y ancianos, internados en el Hospital São Marcos, en Teresina (PI). Los pacientes se sometieron a la evaluación nutricional por antropometría clásica (\% PP, CB, CMB, AMBc y el PCT) y a la medida de la espesura del músculo aductor del pulgar. El estado nutricional de los pacientes se caracteriza por la pérdida grave de peso $(56,1 \%)$, la preservación de la masa muscular y la prevalencia de pérdida grave de la masa grasa $(25,93 \%)$. El promedio de la espesura del músculo aductor del pulgar para el total de la muestra fue de $13 \pm 3,2 \mathrm{~mm}$. El músculo aductor del pulgar no se asoció con el período de hospitalización y la mortalidad. El \% PP fue el parámetro que mejor se correlacionó con el período de hospitalización ( $\mathrm{p}=0,007)$. Los demás parámetros no se asociaron a ninguno de los indicadores de morbimortalidad estudiados. La espesura del músculo aductor del pulgar no ha demostrado ser un buen indicador pronóstico antropométrico y esta declaración se puede atribuir a la presencia de un menor comprometimiento del estado nutricional observada en la mayoría de los pacientes y por permanecieren menos tiempo en el hospital.

Palabras clave: Pacientes Internos; Evaluación Nutricional; Antropometría; Estudios Transversales; Análisis Cuantitativo; Teresina (PI) 